\title{
Identification of novel biomarkers for preeclampsia on the basis of differential expression network analysis
}

\author{
YUFANG WU ${ }^{1}$, XIUHUA FU ${ }^{2}$ and LIN WANG $^{3}$ \\ Departments of ${ }^{1}$ Gynecology and ${ }^{2}$ Obstetrics, Binzhou People's Hospital, \\ Binzhou, Shandong 256610; ${ }^{3}$ Department of Obstetrics and Gynecology, Xijing Hospital, \\ The First Affiliated Hospital of The Fourth Military Medical University, Xi'an, Shanxi 710032, P.R. China
}

Received June 12, 2015; Accepted February 11, 2016

DOI: $10.3892 /$ etm.2016.3261

\begin{abstract}
Preeclampsia (PE) is a severe pregnancy complication, which is a leading cause of maternal and fetal mortality. The present study aimed to screen potential biomarkers for the diagnosis and prediction of $\mathrm{PE}$ and to investigate the underlying mechanisms of PE development based on the differential expression network (DEN). The microarray datasets E-GEOD-6573 and E-GEOD-48424 were downloaded from the European Bioinformatics Institute database. Differentially expressed genes (DEGs) between the PE and normal groups were screened by Significant Analysis of Microarrays with the cutoff value of a $\log 2$ fold changel of $>2$, and a false discovery rate of $<0.05$. The DEN was constructed based on the differential and non-differential interactions observed. In addition, genes with higher connectivity degrees in the DEN were identified on the basis of centrality analysis, while disease genes were also extracted from the DEN. In order to understand the functional roles of genes in DEN, Gene Ontology (GO) and pathway enrichment analyses were performed. The present results indicated that a total of 225 genes were considered as DEGs in the PE group, while 466 nodes and 314 gene interactions were involved in the DEN. Among these 466 nodes, 4 nodes with higher degrees were identified, including ubiquitin $\mathrm{C}(U B C)$, small ubiquitin-like modifier 1 (SUMO1), SUMO2 and RAD21 homolog (S. pombe) (RAD21). Notably, $U B C$ was also found to be a disease gene. UBC, RAD21, SUMO2 and SUMO1 were markedly enriched in the regulation of programmed cell death, as well as in the regulation of apoptosis, cell cycle and chromosomal part. In conclusion, based on these results, we suggest that $U B C, R A D 21, S U M O 2$ and SUMO1 may be reliable biomarkers for the prediction of the development and progression of PE.
\end{abstract}

Correspondence to: Mr. Lin Wang, Department of Obstetrics and Gynecology, Xijing Hospital, The First Affiliated Hospital of The Fourth Military Medical University, 127 Changle West Road, Xi'an, Shanxi 710032, P.R. China

E-mail: wanglin053476@sina.com

Key words: preeclampsia, differentially expressed genes, differential expression network, disease gene

\section{Introduction}

Preeclampsia (PE) is a severe pregnancy complication, characterized by hypertension and large amounts of urine protein (1). $\mathrm{PE}$ increases the risk of red blood cell breakdown, impaired liver function and kidney dysfunction $(2,3)$. According to the World Health Organization, approximately $2-8 \%$ of pregnancies are affected by PE worldwide (4). Additionally, $\sim 29,000$ cases of mortality were reported in 2013 (5). However, a specific treatment for PE and methods for its early diagnosis or prediction have not been adequately developed. Therefore, understanding the molecular mechanisms underlying PE is essential.

PE is know to be associated with the dysregulation of susceptibility genes. Recently, the molecular mechanism and therapy of PE has been investigated in a number of studies. For instance, Yong et al (6) have demonstrated that several susceptibility genes, including inhibin $\beta \mathrm{A}$, angiotensinogen, interleukin-6, interferon and transforming growth factor $\beta 1$ genes, serve an important role in the development and progression of PE through apoptosis and cell signaling. Similarly, expression of fms-like tyrosine kinase-1 and kinase insert domain containing receptor has been reported to contribute to the pathogenesis of PE $(7,8)$. A previous study has also indicated that the increased level of vascular endothelial growth factor may be an important mechanism underlying PE via regulating angiogenesis and blood flow (9). Furthermore, Long et al (10) have suggested that inactivating killer-cell immunoglobulin-like receptors may affect the risk of PE possibly by lowering the activation of uterine natural killer cells. The gene $\mathrm{V}$-set and immunoglobulin domain containing 4 has been identified to be upregulated in the peripheral blood mononuclear cells from PE patients (11). However, PE is a multi-system disorder and its underlying molecular mechanism remains unclear.

Currently, microarray analysis is widely utilized to study the development and progression of various diseases, as well as to determine the underlying biomarkers of diseases, due to the lower expense and advancements in this technique (12). The gene profile dataset E-GEOD-6573 established by Herse et al (13) screened differentially expressed genes (DEGs) from $\mathrm{PE}$ and control tissue samples, based on the presence of a 4-fold change in gene expression. The identification of DEGs and Gene Ontology (GO) functional annotation of PE and 
control samples are also performed in the gene profile dataset of E-GEOD-48424 provided by Textoris et al (14).

In the present study, the differential expression network (DEN) strategy was employed to trace the dysfunctional interactions associated with the development and progression of PE (15). DEN is a novel network that includes differential genes and networks, and also covers non-differential interactions associated with the disease, which are not considered in the differential network (15). In order to obtain further insight into the mechanism underlying PE development and progression, two gene expression microarray datasets of PE were downloaded from the European Bioinformatics Institute (EMBL-EBI) database and merged, followed by the identification of DEGs. In addition, DENs were constructed by screening the differential interactions and non-differential interactions. Subsequently, hub genes and disease genes were extracted from the DEN. GO and pathway enrichment analysis were also conducted for the genes in DEN. The results suggest that hub genes and disease genes identified in the present study provide a theoretical basis for the treatment of PE.

\section{Materials and methods}

Microarray data. In total, two microarray datasets were downloaded from the EMBL-EBI database, including the E-GEOD-6573 (13) and E-GEOD-48424 datasets (14). Gene expression data from E-GEOD-6573, containing abdominal adipose, muscle and placenta samples from $10 \mathrm{PE}$ women and 10 women with uneventful pregnancies, were obtained using the GPL570 platform of Affymetrix Human Genome U133 Plus 2.0 Array (Affymetrix, Inc., Santa Clara, CA, USA). The microarray data of E-GEOD-48424 included 19 PE samples and 19 normal pregnancy tissue samples and were obtained based on the GPL6480 platform of Agilent-014850 Whole Human Genome Microarray 4x44 K G4112F (Agilent Technologies, Santa Clara, CA, USA).

Data preprocessing. Prior to analysis, the 'expresso' function of the Affy package (16) was used to preprocess the gene profile data of E-GEOD-6573. The specific steps of the preprocessing were as follows: The 'rma' function (17) was applied to perform background correction, and then normalization was performed using the quartile function in order to eliminate the influence of nonspecific hybridization (18). Subsequently, the perfect match probe correction was performed via MAS (19), followed by the expression summary through median polish. AffyBatch data were converted into expression measurements and the 'featureFilter' function was then utilized to filter the data for removing the redundant and irrelevant features. Finally, the probe sets were aligned to the genes using the 'getSymbol' function.

Simultaneously, processed data and the gene annotation file of E-GEOD-48424 were downloaded. Subsequently, the probe sets were mapped to the genes using the 'getSymbol' function in Package 'annotate' (version 1.49.1; http://www.bioconductor.org/).

Identification of DEGs in PE. In the analysis performed in the present study, the merge function from Package 'inSilicoMerging' (version 1.10.1; https://www.bioconductor.
org/packages/release/bioc/html/inSilicoMerging.html) was used to merge the two microarray datasets into one global dataset, in order to further obtain a merged data set by means of the geNorm normalization method (20). Next, Significant Analysis of Microarrays (R package; version 1.25; https://www.r-project.org/) was used to identify DEGs in the PE samples relative to normal samples (21). The Benjamini-Hochberg approach (22) was employed to adjust the raw P-value into the false discovery rate (FDR). Several genes were considered as DEGs when the llog2 fold changel was $>2$, and the FDR was $<0.05$.

Construction of protein-protein interactions (PPI) network. Initially, all human PPIs, involving 15,750 genes and 248,584 interactions were downloaded from the Biological General Repository for Interaction Datasets (BioGrid; http://thebiogrid.org/) database. Subsequently, all genes of the two microarray datasets used in the present study were aligned to the compiled PPI network to filter several unnecessary interactions. A total of 9,427 genes with 151,836 interactions were selected.

Spearman's correlation coefficient calculation. In the current study, Spearman's correlation coefficient was calculated to determine the interactions among genes. Subsequent to obtaining gene expression values between normal and PE samples, Spearman's correlation coefficient was calculated for the 151,836 interactions in different conditions, which were represented by the normal and PE samples, named as A1 and A2, respectively. Similarly, the absolute value of the difference in the Spearman's correlation coefficient between the two groups, defined as |A1-A2I, was also calculated.

Construction of DEN. Two PPI models were randomly constructed (one for normal samples and the other for PE samples) to select gene interactions for subsequent analysis, with 500,000 gene interactions present in each model. Next, the Spearman's correlation coefficients of the interactions in the two PPI models (A1 and A2) were computed, and |A1-A2| was also calculated. Subsequently, |A1-A2| was ranked in descending order, and |A1-A2I was found to be 0.548 when the $\mathrm{P}$ value was 0.05 . A differential interaction was considered in cases where the IA1-A2I value was $>0.548$ and at least one of $\mathrm{A} 1$ and $\mathrm{A} 2$ was $>0.7$. By contrast, if the $|\mathrm{A} 1-\mathrm{A} 2|$ value of a gene interaction was $\leq 0.548$ and two corresponding nodes (linked genes or coded proteins) were both DEGs, the edge was regarded as a non-differential interaction. The DEN was then constructed by incorporating all the differential and non-differential interactions.

Identifying the disease genes associated with PE in the DEN. The PE-associated genes (regarded as disease genes) were obtained from the GeneCards database (www.genecards.org). Only those genes whose expressions were examined in the DEN were selected in the present study.

Centrality analysis. The measures of centrality are broadly applied in network analysis, and include the degree, closeness, betweenness and Eigenvector centrality (23). Among these, the degree is the simplest indicator of centrality. Degree is defined 
Table I. Genes with the top $1 \%$ degrees of centrality in the differential expression network.

\begin{tabular}{lc} 
Gene symbol & Centrality degree \\
\hline$U B C$ & 29 \\
SUMO2 & 5 \\
SUMO1 & 5 \\
RAD21 & 5 \\
\hline
\end{tabular}

$U B C$, ubiquitin C; $S U M O$, small ubiquitin-like modifier; $R A D 21$, RAD21 homolog (S. pombe).

as the number of links that a node has with other nodes (24). In the present study, the degree distribution was examined and the nodes with the top $1 \%$ degrees of centrality were identified as the hub genes.

Functional enrichment analysis for the genes in DEN. The GO database (www.geneontology.org) frequently provides biological information on large-scale genes (25). In addition, the Kyoto Encyclopedia of Genes and Genomes (KEGG; www. genome.jp/kegg) is a bioinformatics database that includes a variety of biochemistry pathways (26), while the Database for Annotation, Visualization and Integrated Discovery (DAVID; http://david.abcc.ncifcrf.gov/) is an analytic tool used in the determination of the biological meaning for a large number of genes (27). In the current study, DAVID was applied for GO functional annotation and KEGG pathway enrichment analysis of genes in the DEN. The expression analysis systematic explorer test was applied to assess the significant categories. Significant enrichment was determined based on the presence of at least two target genes and a P-value of $<0.01$ in the GO and pathways.

\section{Results}

Microarray data analysis. A total of 20,102 and 18,411 genes were identified in the gene expression data of E-GEOD-6573 and E-GEOD-48424, respectively. Furthermore, a total of 11,269 genes were obtained subsequent to merging. Microarray analysis identified 225 genes as DEGs, including 9 upregulated and 216 downregulated genes.

DEN construction. The distribution of Spearman's correlation coefficient of interactions for the normal and PE conditions is exhibited in Fig. 1. The mean Spearman's correlation coefficients were found to be 0.0688 and 0.0640 in the normal and PE groups, respectively. A decrease was identified in Spearman's correlation coefficient distribution (0.1-0.6) of 33,653 interactions in the PE network relative to 35,035 interactions in the normal network. By contrast, an increase in Spearman's correlation coefficient distribution (-0.2 to 0.1$)$ of interactions in PE $(29,019$ interactions) relative to those in the normal network $(28,406$ interactions) was observed. Next, the |A1-A2| distribution of interactions for these two groups was calculated (Fig. 2). A total of 283 differential interactions were identified, with a |A1-A2I

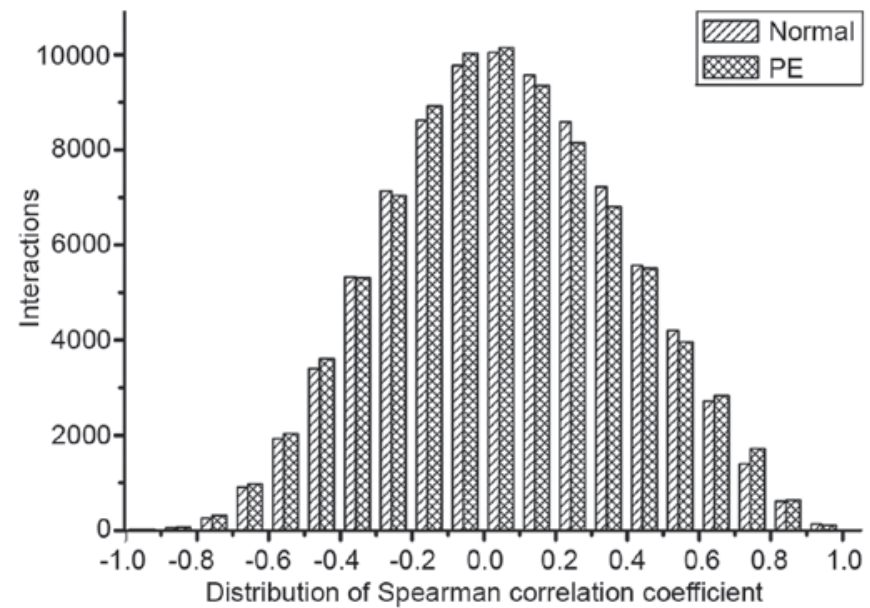

Figure 1. Distribution of Spearman's correlation coefficient distribution of interactions in the normal and PE groups. PE, preeclampsia.
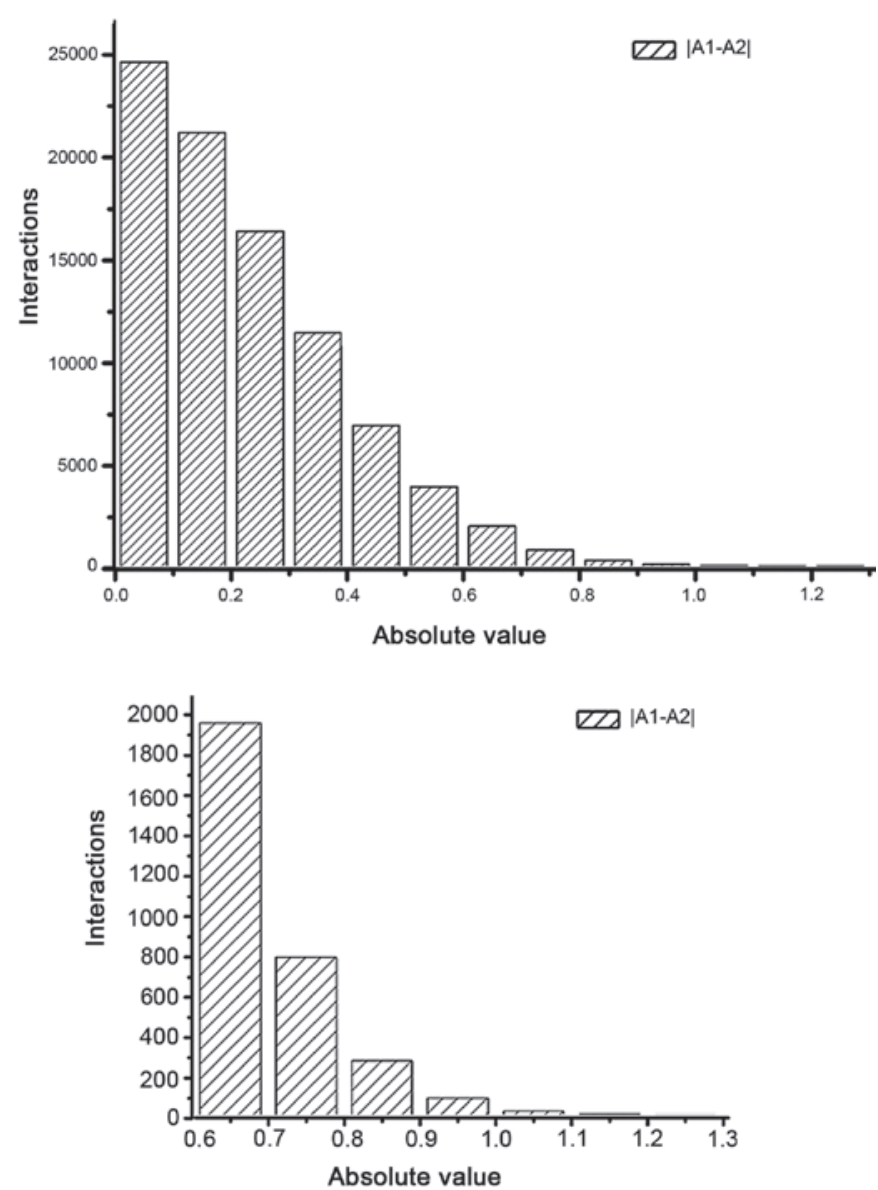

Figure 2. Distribution of absolute value of the difference of Spearman's correlation coefficient between normal (A1) and PE (A2) groups, shown as |A1-A2I. Top panel, distribution between absolute values 0.0 and 1.3; bottom panel, distribution between absolute values 0.6 and 1.3. PE, preeclampsia.

of $>0.548$, as well as at least one of the A1 and A2 values being $>0.7$. Furthermore, 31 non-differential interactions were observed in edges with |A1-A2| of $\leq 0.548$ and DEGs in two corresponding nodes. Hence, a total of 466 nodes and 314 gene interactions were involved in the DEN. Fig. 3 shows the detailed main network. 


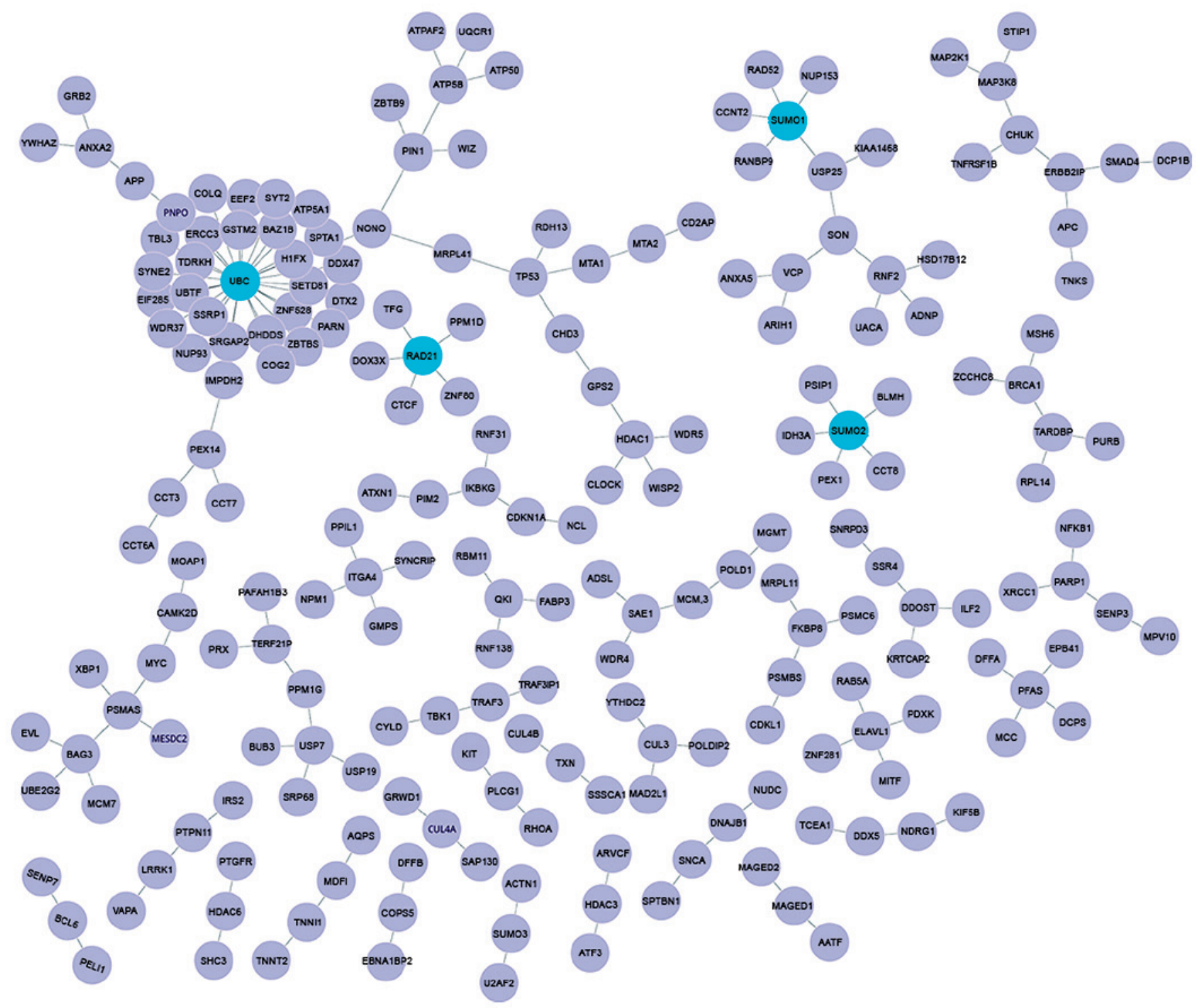

Figure 3. Construction of differential expression network involving 466 nodes and 314 gene interactions. Purple nodes represent the genes, the edges represent the association of the genes, and the light blue nodes represent the hub genes (UBC, RAD21, SUMO1 and SUMO2).

Identifying the disease genes associated with PE in DEN. In total, 20 disease genes with 55 differential interactions were identified. Among those 20 disease genes, ubiquitin C $(U B C)$ was found to possess the highest connectivity degree (degree, 29).

Centrality analysis. Among the 466 nodes in the DEN, 4 nodes presented centrality degrees in the top $1 \%$, as shown in Table I. These nodes involved the following genes: $U B C$ (degree, 29), small ubiquitin-like modifier 2 (SUMO2; degree, 5), SUMO1 (degree, 5), RAD21 homolog (S. pombe) (RAD21; degree, 5).

GO functional annotation and KEGG enrichment analysis of genes in the DEN. Based on the presence of $<2$ target genes and $\mathrm{P}<0.01$, as shown in Table II, the significant functions in the biological process (BP) term of GO included negative regulation of macromolecule metabolic process, regulation of programmed cell death, regulation of apoptosis and cell cycle, while significant functions in the cellular component (CC) term included the chromosomal part, nuclear and organelle lumen. More specifically, $U B C$, a disease and hub gene, was significantly involved in the regulation of programmed cell death, as well as in the regulation of apoptosis in the BP. Genes such as SUMO2 and SUMO1 were mainly involved in the chromosome and chromosomal part in the CC.

Notably, 27 pathways were obtained using KEGG analysis. The top 10 pathways are presented in Table III. Among these, the most significant pathway was found to be chronic myeloid leukemia. Other significant pathways included the neurotrophin signaling pathway, pathways in cancer and cell cycle. DEGs, including RAD21, participated in the determined pathway, such as in the cell cycle.

\section{Discussion}

In order to clarify the molecular mechanisms underlying PE development, we comprehensively analyzed the gene expression profiles in the E-GEOD-6573 and E-GEOD-48424 datasets using DEN analysis. A total of 225 DEGs were selected from the PE samples, and the hub genes were found to 
Table II. Top $10 \mathrm{BP}$ and CC terms of GO functional annotation of genes in the differential expression network.

\begin{tabular}{|c|c|c|c|}
\hline Term & Term name & Count & P-value \\
\hline GO-BP & Negative regulation of macromolecule metabolic process & 72 & $2.32 \times 10^{-18}$ \\
\hline GO-BP & Regulation of programmed cell death & 74 & $4.07 \times 10^{-17}$ \\
\hline GO-BP & Regulation of cell death & 74 & $4.96 \times 10^{-17}$ \\
\hline GO-BP & Regulation of apoptosis & 73 & $8.50 \times 10^{-17}$ \\
\hline GO-BP & Cell cycle & 66 & $1.09 \times 10^{-13}$ \\
\hline GO-BP & Ubiquitin-dependent protein catabolic process & 34 & $5.84 \times 10^{-13}$ \\
\hline GO-BP & Response to DNA damage stimulus & 41 & $5.26 \times 10^{-12}$ \\
\hline GO-BP & Negative regulation of molecular function & 38 & $1.33 \times 10^{-11}$ \\
\hline GO-BP & Cellular response to stress & 51 & $1.53 \times 10^{-11}$ \\
\hline GO-BP & Negative regulation of nucleobases & 48 & $1.77 \times 10^{-11}$ \\
\hline $\mathrm{GO}-\mathrm{CC}$ & Nuclear lumen & 125 & $1.01 \times 10^{-30}$ \\
\hline $\mathrm{GO}-\mathrm{CC}$ & Organelle lumen & 140 & $9.76 \times 10^{-30}$ \\
\hline $\mathrm{GO}-\mathrm{CC}$ & Intracellular organelle lumen & 138 & $1.31 \times 10^{-29}$ \\
\hline $\mathrm{GO}-\mathrm{CC}$ & Membrane-enclosed lumen & 140 & $7.65 \times 10^{-29}$ \\
\hline $\mathrm{GO}-\mathrm{CC}$ & Nucleoplasm & 90 & $1.82 \times 10^{-26}$ \\
\hline $\mathrm{GO}-\mathrm{CC}$ & Non-membrane-bound organelle & 151 & $1.29 \times 10^{-19}$ \\
\hline $\mathrm{GO}-\mathrm{CC}$ & Intracellular non-membrane-bound organelle & 151 & $1.29 \times 10^{-19}$ \\
\hline $\mathrm{GO}-\mathrm{CC}$ & Chromatin remodeling complex & 43 & $3.72 \times 10^{-11}$ \\
\hline $\mathrm{GO}-\mathrm{CC}$ & Chromosomal part & 37 & $5.73 \times 10^{-10}$ \\
\hline $\mathrm{GO}-\mathrm{CC}$ & Ribonucleoprotein complex & 42 & $4.05 \times 10^{-9}$ \\
\hline
\end{tabular}

$\mathrm{BP}$, biological process; CC, cellular component; GO, gene ontology.

Table III. Top 10 KEGG pathways of genes in the differential expression network.

\begin{tabular}{lcc}
\hline Term name & Count & P-value \\
\hline Chronic myeloid leukemia & 19 & $7.04 \times 10^{-10}$ \\
Neurotrophin signaling pathway & 22 & $2.22 \times 10^{-8}$ \\
Pathways in cancer & 35 & $3.64 \times 10^{-7}$ \\
Acute myeloid leukemia & 14 & $4.27 \times 10^{-7}$ \\
Prostate cancer & 16 & $2.67 \times 10^{-6}$ \\
Cell cycle & 19 & $2.81 \times 10^{-6}$ \\
Spliceosome & 19 & $3.16 \times 10^{-6}$ \\
Adipocytokine signaling pathway & 13 & $1.45 \times 10^{-5}$ \\
RIG-I-like receptor signaling pathway & 13 & $2.67 \times 10^{-5}$ \\
ErbB signaling pathway & 14 & $4.79 \times 10^{-5}$ \\
\hline
\end{tabular}

KEGG, Kyoto Encyclopedia of Genes and Genomes.

be $U B C, R A D 21, S U M O 2$ and SUMO1, since they had higher connectivity degrees in the DEN. Furthermore, $U B C, R A D 21$, $S U M O 2$ and SUMO1 were markedly enriched in the regulation of programmed cell death, as well as in the regulation of apoptosis, cell cycle and chromosomal part.

The molecular network is characterized by the intricate interactions that regulate gene expression and cellular functions, thus playing an important role in disease development and progression $(28,29)$. The PPI and gene regulatory networks have been developed to identify available genes on the basis of biomolecular networks $(30,31)$. Nevertheless, several genes may be ignored if their expression is not found to be highly associated across the entire dataset (32). On the contrary, DEN is a novel network that not only includes differential genes and networks, but also covers non-differential interactions associated with disease, which are not considered in differential networks (14). Furthermore, a previous study has demonstrated that DEN analysis may obtain 3-4 times more known disease genes compared with the traditional DEG method (15). For instance, the disease gene $U B C$ identified in the current study was not a DEG, and thus would have not been identified by the traditional DEG method. Accordingly, the present results suggest that DEN can fully extract disease genes and interactions more accurately.

In the present study, we found that $U B C$ was a disease gene, and was involved in the regulation of programmed, cell death, as well as in the regulation of apoptosis in the BP of GO. It is well documented that increased trophoblast cell apoptosis is a typical characteristic of PE placenta $(33,34)$. Dysregulation of ubiquitin-proteasome system is related with the gestational trophoblast disorder $(35,36)$. Notably, the UBC gene encodes protein products required to further generate free ubiquitin in eukaryotes (37). Furthermore, Kugawa and Aoki (37) have reported that UBC promoter regulates to various types of stress, for example, pro-apoptotic stimulus. Thus, we hypothesize that the $U B C$-mediated apoptotic mechanism of $\mathrm{PE}$ through regulation of the ubiquitin-proteasome system is greatly significant.

The abnormal placenta formation is known to be the first stage of PE (38), and placental trophoblast cells have abnormal 
cell cycle mechanisms (39). Unek et al (40) have also indicated that placental alterations of PE may be connected to the cell cycle arrest. In the current study, the cell cycle pathway was identified, which is an important pathway, involving the RAD21 gene that was downregulated. RAD21, as a subunit of a cohesion complex, holds sister chromatids together during the late stage of cell division. Notably, the cohesion of sister chromatid in the period of DNA replication serves a crucial role in the cell cycle of eukaryote (41). Furthermore, Wong and Blobel have indicated that $R A D 21$ is localized at mitotic spindles (42), whereas another study has demonstrated that $R A D 21$ serves a vital role in the transition of phase S to G2 (43). Similarly, in an RNA-sequencing analysis associated with colorectal cancer, the underexpression of $R A D 21$ was found to impair the assembly of spindle or delay the progression of the $S$ phase $(44,45)$. Based on the aforementioned results, it is suggested that RAD21 may affect the risk of PE development through the regulation of cell cycle.

SUMO2 and SUMOI are two members of SUMO proteins, which are small ubiquitin-associated modifiers and regulate multiple cellular processes including DNA repair (46). Chromosomal DNA damage in pregnancy may be a basic pathological feature of PE, and reducing DNA damage may improve the health of the mother and the baby (47). In addition, the changes of placental SUMOylation pathway and free SUMOs may contribute to the etiopathogenesis of severe PE due to abnormal expression of $U B C 9$ (48). Emerging evidence indicated that $U B C 9$-mediated SUMOylation is helpful in maintaining the genome integrity of replicating chromosomes (49). Consistent with this observation, the function annotation exhibited that SUMO2 and SUMO1 were significantly enriched in the chromosome and chromosomal part GO terms. In light of all the aforementioned findings, it can be concluded that SUMO2 and SUMOI may serve an important role in PE progression via the aforementioned functions.

In conclusion, UBC, RAD21, SUMO2, SUMO1 and their enriched functions in the regulation of programmed cell death, regulation of apoptosis, cell cycle and chromosomal part may exert important roles in the development and progression of PE. Therefore, they may be employed as potential therapeutic target in the treatment of PE and enhance the clinical therapeutic efficacy in the future. Nevertheless, these hypotheses require confirmation using animal experiments in further studies.

\section{References}

1. Eiland E, Nzerue C and Faulkner M: Preeclampsia 2012. J Pregnancy 2012: 586578, 2012.

2. Al-Jameil N, Aziz Khan F, Fareed Khan M and Tabassum H: A brief overview of preeclampsia. J Clin Med Res 6: 1-7, 2014.

3. American College of Obstetricians and Gynecologists; Task Force on Hypertension in Pregnancy: Hypertension in pregnancy. Report of the American college of obstetricians and gynecologists' task force on hypertension in pregnancy. Obstet Gynecol 122: 1122-1131, 2013.

4. World Health Organization: WHO recommendations for prevention and treatment of preeclampsia and eclampsia. World Health Organization, Geneva, 2011.

5. GBD 2013 Mortality and Causes of Death Collaborators: Global, regional and national age-sex specifi c all-cause and cause-specifi c mortality for 240 causes of death, 1990-2013: A systematic analysis for the Global Burden of Disease Study 2013. The Lancet 385: 117-171, 2015.
6. Yong HE, Melton PE, Johnson MP, Freed KA, Kalionis B, Murthi P, Brennecke SP, Keogh RJ and Moses EK: [81-OR]: Genome-wide transcriptome directed pathway analysis of maternal preeclampsia susceptibility genes. Pregnancy Hypertens 5: 43-44, 2015.

7. Munaut C, Lorquet S, Pequeux C, Coulon C, Le Goarant J, Chantraine F, Noël A, Goffin F, Tsatsaris V, Subtil D and Foidart JM: Differential expression of VEGFR-2 and its soluble form in preeclampsia. PloS One 7: e33475, 2012.

8. Tripathi R, Rath G, Jain A and Salhan S: Soluble and membranous vascular endothelial growth factor receptor-1 in pregnancies complicated by pre-eclampsia. Ann Anat 190: 477-489, 2008.

9. Sundrani DP, Reddy US, Joshi AA, Mehendale SS, Chavan-Gautam PM, Hardikar AA, Chandak GR and Joshi SR: Differential placental methylation and expression of VEGF, FLT-1 and KDR genes in human term and preterm preeclampsia. Clin Epigenetics 5: 6, 2013.

10. Long W, Shi Z, Fan S, Liu L, Lu Y, Guo X, Rong C, Cui X and Ding H: Association of maternal KIR and fetal HLA-C genes with the risk of preeclampsia in Chinese Han population. Placenta 36: 433-437, 2015.

11. Sun CJ, Zhang L and Zhang WY: Gene expression profiling of maternal blood in early onset severe preeclampsia: Identification of novel biomarkers. J Perinat Med 37: 609-616, 2009.

12. Nakanishi T, Oka T and Akagi T: Recent advances in DNA microarrays. Acta Med Okayama 55: 319-328, 2001.

13. Herse F, Dechend R, Harsem NK, Wallukat G, Janke J, Qadri F, Hering L, Muller DN, Luft FC and Staff AC: Dysregulation of the circulating and tissue-based renin-angiotensin system in preeclampsia. Hypertension 49: 604-611, 2007.

14. Textoris J, Ivorra D, Ben Amara A, Sabatier F, Ménard JP, Heckenroth H, Bretelle F and Mege JL: Evaluation of current and new biomarkers in severe preeclampsia: A microarray approach reveals the VSIG4 gene as a potential blood biomarker. PloS One 8: e82638, 2013.

15. Sun SY, Liu ZP, Zeng T, Wang Y and Chen L: Spatio-temporal analysis of type 2 diabetes mellitus based on differential expression networks. Sci Rep 3: 2268, 2013.

16. Gautier L, Cope L, Bolstad BM and Irizarry RA: Affy-analysis of Affymetrix GeneChip data at the probe level. Bioinformatics 20: 307-315, 2004.

17. Irizarry RA, Hobbs B, Collin F, Beazer-Barclay YD, Antonellis KJ, Scherf U and Speed TP: Exploration, normalization, and summaries of high density oligonucleotide array probe level data. Biostatistics 4: 249-264, 2003.

18. Bolstad BM, Irizarry RA, Astrand M and Speed TP: A comparison of normalization methods for high density oligonucleotide array data based on variance and bias, Bioinformatics 19: 185-193, 2003.

19. Pepper SD, Saunders EK, Edwards LE, Wilson C and Miller CJ: The utility of MAS5 expression summary and detection call algorithms. BMC Bioinformatics 8: 273, 2007.

20. Taminau J, Meganck S, Lazar C, Steenhoff D, Coletta A, Molter C, Duque R, de Schaetzen V, Weiss Solís DY, Bersini H and Nowé A: Unlocking the potential of publicly available microarray data using inSilicoDb and inSilicoMerging R/Bioconductor packages. BMC Bioinformatics 13: 335, 2012.

21. Tusher VG, Tibshirani R and Chu G: Significance analysis of microarrays applied to the ionizing radiation response. Proc Natl Acad Sci USA 98: 5116-5121, 2001.

22. Benjamini Y and Hochberg Y: Controlling the false discovery rate: A practical and powerful approach to multiple testing. J R Stat Soc Series B Stat Methedol 57: 289-300, 1995.

23. Borgatti SP: Centrality and AIDS. Connections 18: 112-115, 1995.

24. Otte E and Rousseau R: Social network analysis: A powerful strategy, also for the information sciences. J Info Sci 28: 441-453, 2002 .

25. Ashburner M, Ball CA, Blake JA, Botstein D, Butler H, Cherry JM, Davis AP, Dolinski K, Dwight SS, Eppig JT, et al: Gene ontology: Tool for the unification of biology. The gene ontology consortium. Nat Genet 25: 25-29, 2000.

26. Kanehisa M and Goto S: KEGG: Kyoto encyclopedia of genes and genomes. Nucleic Acids Res 28: 27-30, 2000.

27. Huang da W, Sherman BT and Lempicki RA: Systematic and integrative analysis of large gene lists using DAVID bioinformatics resources. Nat Protoc 4: 44-57, 2009.

28. He D, Liu ZP, Honda M, Kaneko S and Chen L: Coexpression network analysis in chronic hepatitis $\mathrm{B}$ and $\mathrm{C}$ hepatic lesions reveals distinct patterns of disease progression to hepatocellular carcinoma. J Mol Cell Biol 4: 140-152, 2012.

29. Liu ZP, Wang Y, Zhang XS and Chen L: Network-based analysis of complex diseases. IET Syst Biol 6: 22-33, 2012. 
30. Jeong H, Mason SP, Barabási AL and Oltvai ZN: Lethality and centrality in protein networks. Nature 411: 41-42, 2001.

31. Teichmann SA and Babu MM: Gene regulatory network growth by duplication. Nat Genet 36: 492-496, 2004.

32. de la Fuente A: From 'differential expression' to 'differential networking'-identification of dysfunctional regulatory networks in diseases. Trends Genet 26: 326-333, 2010.

33. Whitley GS, Dash PR, Ayling LJ, Prefumo F, Thilaganathan B and Cartwright JE: Increased apoptosis in first trimester extravillous trophoblasts from pregnancies at higher risk of developing preeclampsia. Am J Pathol 170: 1903-1909, 2007.

34. Longtine MS, Chen B, Odibo AO, Zhong Y and Nelson DM: Villous trophoblast apoptosis is elevated and restricted to cytotrophoblasts in pregnancies complicated by preeclampsia, IUGR, or preeclampsia with IUGR. Placenta 33: 352-359, 2012.

35. Fu JJ, Lin P, Lv XY, Yan XJ, Wang HX, Zhu C, Tsang BK, Yu XG and Wang H: Low molecular mass polypeptide-2 in human trophoblast: over-expression in hydatidiform moles and possible role in trophoblast cell invasion. Placenta 30 : 305-312, 2009.

36. Hass R, and Sohn C: Increased oxidative stress in pre-eclamptic placenta is associated with altered proteasome activity and protein patterns. Placenta 24: 979-984, 2003.

37. Kugawa $\mathrm{F}$ and Aoki M: Expression of the polyubiquitin gene early in the buprenorphine hydrochloride-induced apoptosis of NG108-15 cells. DNA Seq 15: 237-245, 2004.

38. Roberts JMand Hubel CA: The two stage model of preeclampsia: variations on the theme. Placenta 30 (Suppl A): S32-S37, 2009.

39. Heazell AE, Buttle HR, Baker PN and Crocker IP: Altered expression of regulators of caspase activity within trophoblast of normal pregnancies and pregnancies complicated by preeclampsia. Reprod Sci 15: 1034-1043, 2008.
40. Unek G, Ozmen A, Mendilcioglu I, Simsek M and Korgun ET: The expression of cell cycle related proteins PCNA, Ki67, p27 and p57 in normal and preeclamptic human placentas. Tissue Cell 46: 198-205, 2014.

41. Atienza JM, Roth RB, Rosette C, Smylie KJ, Kammerer S, Rehbock J, Ekblom J and Denissenko MF: Suppression of RAD21 gene expression decreases cell growth and enhances cytotoxicity of etoposide and bleomycin in human breast cancer cells. Mol Cancer Ther 4: 361-368, 2005.

42. Wong RW and Blobel G: Cohesin subunit SMC1 associates with mitotic microtubules at the spindle pole. Proc Natl Acad Sci USA 105: 15441-15445, 2008.

43. Birkenbihl RP and Subramani S: The $\operatorname{rad} 21$ gene product of Schizosaccharomyces pombe is a nuclear, cell cycle-regulated phosphoprotein. J Biol Chem 270: 7703-7711, 1995.

44. Guillou E, Ibarra A, Coulon V, Casado-Vela J, Rico D, Casal I, Schwob E, Losada A and Méndez J: Cohesin organizes chromatin loops at DNA replication factories. Genes Dev 24: 2812-2822, 2010.

45. Kong X, Ball AR Jr, Sonoda E, Feng J, Takeda S, Fukagawa T, Yen TJ and Yokomori K: Cohesin associates with spindle poles in a mitosis-specific manner and functions in spindle assembly in vertebrate cells. Mol Biol Cell 20: 1289-1301, 2009.

46. Ulrich HD: SUMO modification: Wrestling with protein conformation. Current Biol 15: R257-R259, 2005.

47. Furness DL, Dekker GA, Hague WM, Khong TY and Fenech MF: Increased lymphocyte micronucleus frequency in early pregnancy is associated prospectively with pre-eclampsia and/or intrauterine growth restriction. Mutagenesis 25: 489-498, 2010

48. Baczyk D, Drewlo S and Kingdom J: Emerging role of SUMOylation in placental pathology. Placenta 34: 606-612, 2013.

49. Branzei D, Sollier J,Liberi G,Zhao X, Maeda D, Seki M,Enomoto T, Ohta K and Foiani M: Ubc9-and mms21-mediated sumoylation counteracts recombinogenic events at damaged replication forks. Cell 127: 509-522, 2006. 